\title{
Importância da assistência de enfermagem no manejo de complicação relacionada ao cateter totalmente implantável
}

\author{
THE IMPORTANCE OF NURSING CARE IN MANAGING COMPLICATIONS RELATED \\ TO FULLY-IMPLANTABLE CATHETERS
}

IMPORTANCIA DE LA ATENCIÓN DE ENFERMERÍA EN EL MANEJO DE COMPLICACIÓN RELACIONADA AL CATÉTER TOTALMENTE IMPLANTABLE

\section{Luciana Mendes Bruzi ${ }^{1}$, Danielle Cabral Mendes ${ }^{2}$}

\section{RESUMO}

Trata-se de relato de experiência com paciente do sexo feminino, 30 anos, com diagnóstico de recaída de Leucemia Mielóide Aguda, submetida à implantação de cateter e que apresentou necrose na ferida cirúrgica. Foram utilizados com sucesso: colagenase, alginato de cálcio e loção oleosa à base de ácidos graxos essenciais. Ficou claro que as complicações relacionadas a cateteres conferem um grande desafio à enfermagem oncológica além de determinarem atraso no tratamento oncológico. A correta tomada de decisão o quanto antes é de extrema importância e evita a retirada precoce do cateter.

\section{DESCRITORES}

Enfermagem oncológica

Cateteres de demora

Cicatrização de feridas

\begin{abstract}
This is an experience report of a thirty-yearold female patient, diagnosed with recurrence of Acute Myeloid Leukemia, submitted to catheter implantation and presenting surgical wound necrosis. The following were used with success: collagenase, calcium alginate and oil lotion with essential fatty acids. It was found that the complications related to catheters are a great challenge in oncology nursing besides implying a delay in the oncological treatment. Making a correct decision as soon as possible is extremely important and avoids removing the catheter too soon.
\end{abstract}

\section{DESCRIPTORS}

Oncologic nursing

Catheters, indwelling

Wound healing

\begin{abstract}
RESUMEN
Se trata de un relato de experiencia con paciente de sexo femenino, treinta años, con diagnóstico de recaída de Leucemia Mieloide Aguda, sometida a la implantación de catéter, que presentó necrosis en la herida quirúrgica. Fueron utilizados con éxito: colagenasa, alginato de calcio y loción oleosa a base de ácidos grasos esenciales. Quedó claro que las complicaciones relacionadas a catéteres representan un gran desafío para la enfermería oncológica, además de determinar atraso en el tratamiento oncológico. La correcta toma de decisión y la inmediatez son de extrema importancia y evitan el retirado precoz del catéter.
\end{abstract}

\section{DESCRIPTORES}

Enfermería oncológica

Catéteres de permanência

Cicatrización de heridas

\footnotetext{
${ }^{1}$ Mestranda em Gestão da Saúde pela Universidad Del Mar do Chile. Especialista em Oncologia pela Sociedade Brasileira de Enfermagem Oncológica. Especialista em Auditoria em Sistemas de Saúde pela Faculdade São Camilo. MBA em Recursos Humanos pela Escola Superior Aberta do Brasil. Enfermeira do Centro de Quimioterapia Antiblástica e Imunoterapia Ltda. Santa Luzia, MG, Brasil. lubruzi@gmail.com ${ }^{2}$ Enfermeira Especialista em Oncologia pela Sociedade Brasileira de Enfermagem Oncológica. Especialista em Gestão em Saúde pelo Instituto de Educação Continuada da Pontifícia Universidade Católica de Minas Gerais. Enfermeira do Centro de Quimioterapia Antiblástica e Imunoterapia Ltda. Santa Luzia, MG, Brasil. daniellemendes2004@hotmail.com 


\section{INTRODUÇÃO}

A inserção do cateter totalmente implantado para alguns pacientes representa parte essencial do tratamento oncológico, pois muitas vezes é a única via de acesso da medicação quimioterápica ao organismo. Todo o processo, desde a escolha do cateter mais adequado à anatomia do paciente, exames pré-operatórios, o ato cirúrgico em si e por fim o pós-operatório deve ser cercado de cuidados para que este momento seja o menos agressivo possível para o paciente.

Os cateteres são dispositivos de acesso vascular central, desenvolvidos para permitir o acesso repetido ao sistema vascular para a administração de medicamentos, fluidos intravenosos, soluções de nutrição parenteral e substâncias sanguíneas e também são indicados para a coleta de amostras de sangue ${ }^{(1)}$.

O acesso ao reservatório é efetuado através de inserção de uma agulha percutânea, tipo Huber exclusiva para efetuar punções em reservatórios (portal ou port) dos cateteres totalmente implantáveis. Sua ponta tem um formato que impede a retirada de fragmentos de silicone do dome. As agulhas convencionais ou scalps não são recomendadas para a punção do portal porque o danificam e diminuem a sua vida útil, além do risco de extravasamento de drogas na hora da infusão e o risco de embolização pelo deslocamento de fragmentos ${ }^{(2)}$.

As técnicas cirúrgicas utilizadas para a implantação são: punção, técnica de Seldinger, ou dissecção pelo método de Heimbach e Ivey. A técnica de Seldinger (em sua versão original ou modificada) é a mais utilizada ${ }^{(3-4)}$ A veia subclávia é a primeira escolha quando se indica um acesso venoso central, pois é o sítio com menor risco de trombose e de infecção relacionados aos cateteres quando comparado com as veias femoral e jugular interna(5).

O processo de recuperação cicatricial normal pós-operatório deve seguir as etapas habituais de uma perfeita e coordenada cascata de eventos celulares que podem ser definidas através de etapas: coagulação, inflamação, proliferação, contração e remodelação(6). Mas o processo de cicatrização sofre interferências constantes: localização anatômica, tipo de pele, raça e técnica cirúrgica utilizada. A sutura da ferida constitui um dos mais importantes passos para uma cicatrização segura e eficiente. No entanto quando esta etapa ocorre inadequadamente este processo é quebrado e a cicatrização fica comprometida ${ }^{(7)}$. A ação mecânica exercida no tecido durante a sutura deve permitir um meio adequado para a revitalização da ferida, mas quando a força de tração do fio interfere no processo circulatório, ocorre isquemia local e posteriormente a necrose tecidual. A necrose se caracteriza pela morte celular acidental de uma parte de um organismo ainda vivo(8).
Em uma ferida acometida por área de necrose o primeiro passo é tentar a retirada do tecido desvitalizado este processo é denominado debridamento. O objetivo do debridamento é então o de promover a limpeza da região afetada pela necrose. Os métodos de debridamento utilizados são: o autolítico, cujo processo se dá através de enzimas do próprio organismo, que dissolve a necrose na presença de curativos oclusivos; o enzimático, que utiliza agentes químicos que atuam selecionando o tecido necrótico causando danos mínimos aos tecidos adjacentes; o mecânico que se dá através de força física, como fricção e pinçamento para a remoção do tecido morto; e o debridamento cirúrgico como o próprio nome o caracteriza, faz a excisão da necrose por meio cirúrgico ${ }^{(8-9)}$.

Hoje o tratamento de feridas se baseia em uma atividade multidisciplinar, mas que acrescentou para a enfermagem poder de decisão nas condutas e um vasto meio para demonstrar o saber. Cabe destacar que o sucesso do tratamento depende, dentre outros fatores, da criteriosa escoIha, bem como da adequada utilização dos produtos selecionados $^{(10)}$. Hoje no mercado existem vários tipos de cobertura que atuam como método enzimático de debridamento. Para o caso que será relatado a seguir, três tipos de cobertura foram utilizados: a colagenase, o alginato de cálcio e loção oleosa a base de ácidos graxos essenciais ${ }^{(9)}$.

Colagenase: é uma cobertura enzimática que tem por substrato o colágeno nativo e desnaturalizado. Apresenta-se comercialmente em forma de pomada ou creme, cuja função é remover os restos celulares do tecido necrosado, ou seja, realiza o debridamento químico estimulando, indiretamente, a formação do tecido de granulação e, posteriormente, a reepitelização ${ }^{(10)}$. Sua recomendação se restringe a feridas de pequena extensão.

Alginato de Cálcio: sais do ácido algínico extraídos de algas marrons marinhas grandes, a laminaria, compostas pelos ácidos gulurônico e manurônico, apresentando íons cálcio e sódio incorporados, são industrializadas e usadas para fazer moldagens odontológicas e como material absorvente para curativos cirúrgicos. O sódio presente no exsudato e no sangue interage com o cálcio do curativo de alginato. A troca iônica auxilia no debridamento autolítico, tem alta capacidade de absorção, resulta na formação de um gel que mantém o meio úmido para cicatrização e induz a hemostasia.

Loção oleosa: composta por ácido caprílico, ácido cáprico, ácido láurico, lecitina de soja, vitamina $A$, vitamina $E$, ácido capróico e óleo de girassol (ácido linoléico). Possui a capacidade de modificar reações inflamatórias e imunológicas, acelerando o processo de granulação tecidual ${ }^{(10)}$.

\section{RELATO DE EXPERIÊNCIA}

Paciente FSM, sexo feminino, 30 anos, submetida a tratamento para Leucemia Mielóide Aguda (LMA) de abril de 
1998 a abril de 2001. Em outubro de 2008 foi diagnosticada recidiva tardia da doença e imediata internação para inicio da quimioterapia de indução com esquema de segunda linha com hiper-dose (HD) de citarabina (Ara-c), etoposide (VP-16) e mitoxantrona, administrados em acesso venoso periférico de difícil punção. Durante esta internação evoluiu com flebite, candidíase oral e diarréia e foi necessária a infusão de antibioticoterapia sistêmica (vancomicina, metronidazol, meropenem) e antifúngico (anfotericina B) que provocaram um desgaste ainda mais acentuado da rede venosa. Após a recuperação medular foi solicitada a implantação do cateter de longa permanência que foi realizada em 13/11/2008 e a alta hospitalar foi dada no dia seguinte. A paciente retornou para consulta ambulatorial em 24/11/2008 e durante a inspeção da cicatriz de implantação do cateter, foi visualizada necrose tecidual com cerca de $2 \mathrm{~cm}$, localizada na parte final da sutura e a $1 \mathrm{~cm}$ acima do port. Foi feito contato com o cirurgião responsável pelo implante do cateter que autorizou a punção do cateter e sugeriu curativos diários com solução fisiológica. Após discussão do caso entre a equipe de enfermeiras do ambulatório e o oncologista responsável pela paciente optou-se por adiar a utilização do cateter e tentar recuperar a área lesada. Foi atribuída à equipe de enfermagem total autonomia na condução do caso. A paciente foi colocada a par da situação e concordou com a conduta proposta pelas enfermeiras.

A primeira medida tomada já no dia $24 / 11 / 2008$ foi o debridamento mecânico da maior parte possível de tecido necrosado (Figura 1). A ferida apresentava ainda região de tecido necrosado aderido. Foi utilizada a única cobertura de que a instituição dispunha no momento: colagenase, para iniciar o debridamento químico.

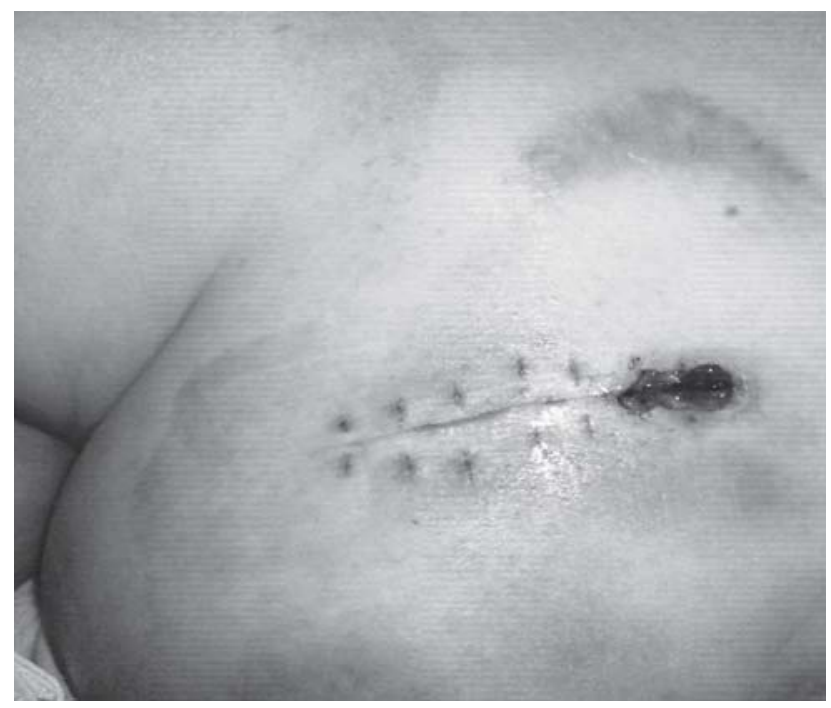

Figura 1 - Necrose tecidual

As trocas de curativo eram diárias e se deu com esta mesma conduta até $28 / 11 / 2008$, quando foi necessário novo debridamento mecânico devido à área macerada (Figura 2).

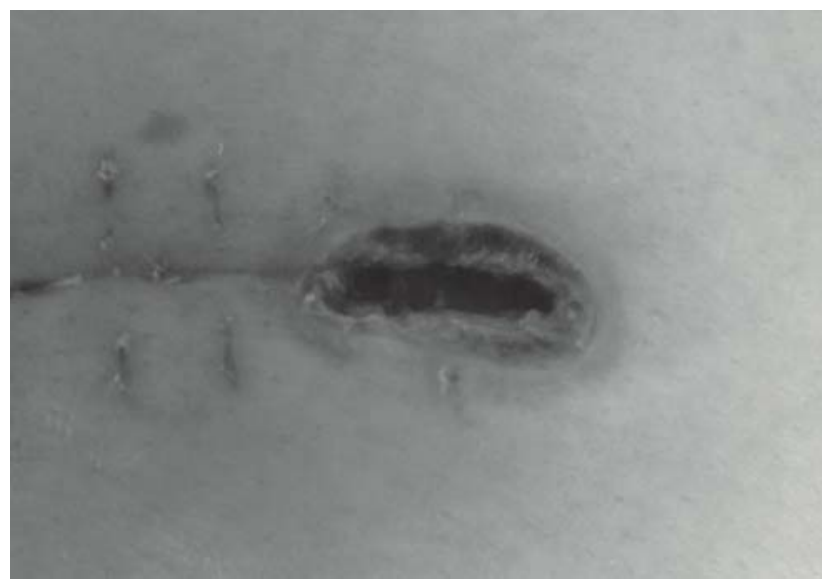

Figura 2 - Presença de tecido macerado

Em 29/11/2008 a cobertura com colagenase foi substituída por alginato de cálcio devido a pouca eficiência. Já no dia 30/11/2008 foi percebida a eficiência da nova cobertura, com a retirada de grande extensão de necrose (Figura 3).

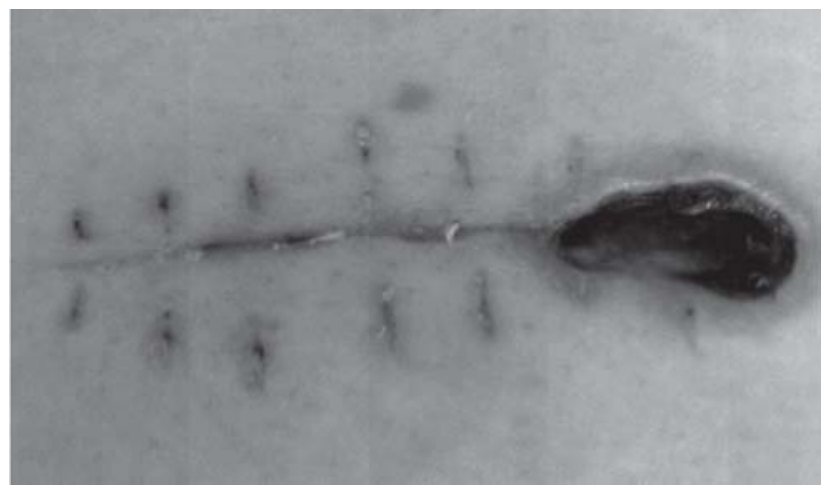

Figura 3 - Debridamento químico lento

Em 03/12/2008 foi avaliado que toda a necrose havia sido retirada restando apenas $1 / 3$ da área com fibrina. Foi mantida a cobertura com alginato de cálcio para a retirada da fibrina. A partir desta data foi iniciada a aplicação de acido linoléico (Dersani ${ }^{\circledR}$ ) nas bordas da ferida com a finalidade de proteger e recuperar a área (Figura 4).

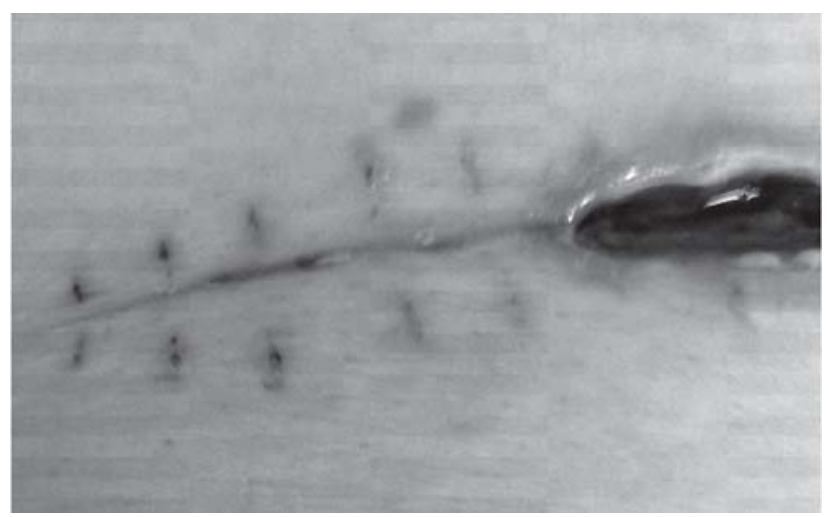

Figura 4 - Ausência de necrose
Rev Esc Enferm USP

2011; 45(2):522-6

www.ee.usp.br/reeusp/
Importância da assistência de enfermagem no manejo de complicação relacionada ao cateter totalmente implantável Bruzi LM, Mendes DC 
Apesar do leito da ferida não ter se recuperado completamente a equipe de enfermagem e o oncologista decidiram que o cateter poderia ser utilizado, mas ainda com o acompanhamento diário. A paciente internou em 05/12/ 2008 para o segundo ciclo de quimioterapia com citarabina por cinco dias e mitoxantrona por dois dias. Devido à proximidade entre o cateter e a lesão optou-se por fazer, apesar da dificuldade, dois curativos independentes evitando assim que o contato da cobertura ou secreção proveniente da ferida contaminasse o cateter. A enfermeira da unidade de internação foi comunicada do caso da paciente e em consenso ficou decidido que a paciente então seria encaminhada diariamente ao ambulatório da quimioterapia para realização dos curativos. A cobertura com alginato de cálcio no leito da lesão, assim como a utilização de Dersani ${ }^{\circledR}$ nas bordas permaneceram até o dia 07/12/2008. A partir de então foi decidido apenas colocação de Dersani ${ }^{\circledR}$ para acelerar o processo de epitelização.

Em 11/12/2008 a paciente recebeu alta hospitalar, mas reafirmou o compromisso de retornar diariamente ao ambulatório para a troca do curativo. A equipe de enfermagem optou por continuar com o acompanhamento rigoroso da ferida, pois após as sessões de quimioterapia a imunidade da paciente ficaria comprometida e o risco de infecção tanto local quanto sistêmica era alto. A ferida apresentava-se em franco processo de granulação (Figura 5).

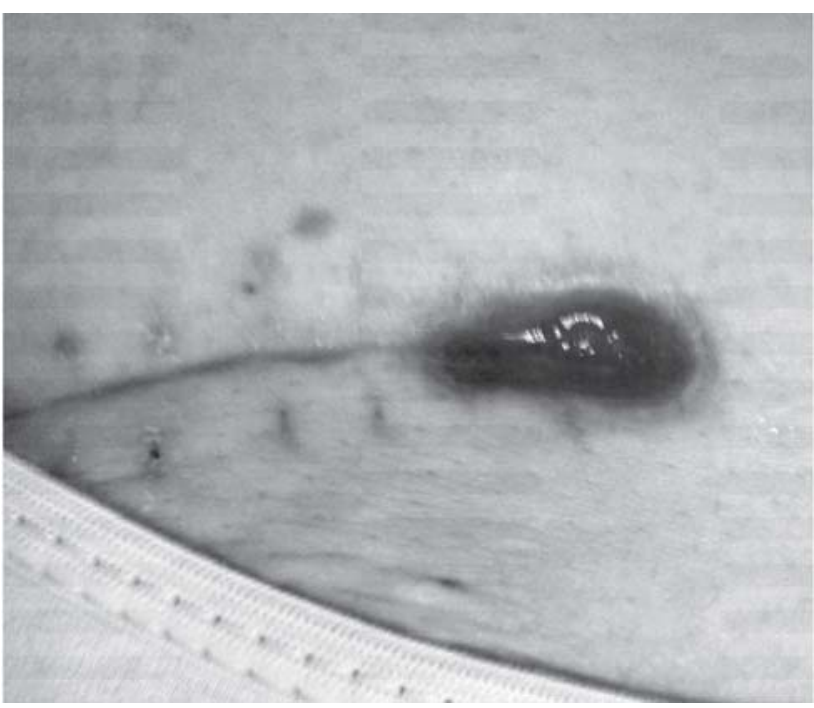

Figura 5 - Franco processo de granulação

A paciente internou no período de 19 a 27/12/2008 devido ao quadro de neutropenia febril. O cateter foi novamente puncionado, desta vez para antibioticoterapia, seguindo as mesmas condutas tomadas anteriormente: dois curativos independentes, mas desta vez utilizando apenas o Dersani ${ }^{\circledR}$ como cobertura.

A partir do dia 28/12/2008 a paciente foi orientada a utilizar o Dersani ${ }^{\circledR}$ diariamente, mas em domicílio até a data do retorno em 07/01/2009. Foi orientada a retornar ao serviço ao mínimo sinal de febre ou mudança na aparência da ferida.
No retorno em 07/01/2008 a paciente foi internada para o terceiro ciclo de quimioterapia: citarabina e etoposide por cinco dias e mais uma vez a conduta de comparecer ao ambulatório diariamente para a troca do curativo foi mantida, assim como a realização de dois curativos independentes: um para o cateter e outro para a ferida que neste período já se apresentava em processo de epitelização. A paciente recebeu alta em 12/01/2008.

\section{DISCUSSÃO}

Para conduzir este caso, alguns pontos foram relevantes na tomada de decisão das enfermeiras. Um deles é o fato de que compete ao profissional enfermeiro, segundo a Deliberação COREN-MG 65/00 atuar tanto na prevenção quanto no tratamento das lesões cutâneas, realizando consulta, prescrevendo e orientando o tratamento, mas para tal, deve-se ter o conhecimento específico sobre a natureza da lesão e viabilidade de tratamento, prezando pelo Código de Ética dos Profissionais de Enfermagem, que segundo a Resolução COFEN 311/2007 estabelece como Responsabilidades e Deveres:

Art. $5^{\circ}$ - Exercer a profissão com justiça, compromisso, equidade, resolutividade, dignidade, competência, responsabilidade, honestidade e lealdade.

Art. $12^{\circ}$ - Assegurar à pessoa, família e coletividade assistência de Enfermagem livre de danos decorrentes de imperícia, negligência ou imprudência.

Art. $13^{\circ}-$ Avaliar criteriosamente sua competência técnica, científica, ética e legal e somente aceitar encargos ou atribuições, quando capaz de desempenho seguro para si e para outro.

Por se tratar de uma clínica oncológica e de um caso inédito, a disponibilidade de recursos materiais para o tratamento de feridas era restrito, então para colocar em prática as intervenções se fez necessário uma avaliação criteriosa tanto destes recursos quanto o aspecto ontológico, conhecendo bem o ser a quem se destinava o cuidado(11-12).

O tratamento se iniciou com a colagenase, pois era a única cobertura disponível com propriedade para realizar o debridamento químico, mas foi imediatamente substituída pelo alginato de cálcio devido às suas propriedades de propiciar o tratamento da ferida em meio úmido, preservando o fluido cicatricial e atuando de forma a auxiliar o debridamento autolítico e induzindo a hemostasia. Outro ponto crucial foi a aderência da paciente ao tratamento com o seu comparecimento diário para a troca dos curativos. O tratamento intercalou momentos de internação, consultas ambulatoriais e tratamento domiciliar. Durante as internações, o contato com a enfermeira responsável pela unidade foi aberto e em consenso, enfermagem e paciente, decidiu-se que toda a responsabilidade pelo tratamento da ferida seria das enfermeiras do ambulatório de quimioterapia. Para que a paciente fizesse as trocas de curativo, diariamente a equipe da unida- 
de de internação encaminhava a paciente ao ambulatório de quimioterapia (que também funciona diariamente). A responsabilidade dos retornos diários, inclusive nos finais de semana, durante as fases de tratamento ambulatorial, foi concedida à paciente após uma avaliação da sua história pregressa, seu contexto social, familiar, cultural, psicológico e espiritual. Com a confiança mútua instituída e após todo o processo educativo onde foi explicado passo-a-passo a técnica a ser utilizada, a paciente adquiriu autonomia para a realização da troca de curativo em domicílio assim que a ferida alcançou a fase de epitelização. Foi instituído então o uso do Dersani ${ }^{\circledR}$ que revitaliza e mantém o equilíbrio hídrico da pele, meIhorando a elasticidade, contribuindo para o restabelecimento da integridade da pele. Logicamente os retornos para a avaliação das enfermeiras eram periódicos até ser estabelecido o fim do tratamento com êxito.

\section{REFERÊNCIAS}

1. Niederhuber JE, Ensminger W, Gyves JW, Liepman M, Doan K, Cozzi E. Totally implanted venous and arterial access system to replace external catheter in cancer treatment. Surgery. 1982;92(4):706-12.

2. Wolosker N, Yazbek G, Nishinari K, Malavolta LC, Munia MA, Langer $\mathrm{M}$, et al. Totally implantable venous catheters for chemotherapy: experience in 500 patients. Sao Paulo Med J. 2004;122(4):147-51.

3. Pinto RD, Lissa FCT, Covatti E, Scheffer LG, Junqueira Júnior G, Sampaio JA. Alternativa técnica para a colocação de cateter totalmente implantável para quimioterapia. Acta Oncol Bras. 1997; 17(1):33-6.

4. Kock HJ, Pietsch M, Krause U, Wilke H, Eigler FW. Implantable vascular access systems: experience in 1500 patients with totally implanted central venous port systems. World J Surg. 1998; 22(1):12-6.

5. Godoy JL, Otta EK, Miyazaki RA, Bitencourt MA, Pasquini R. Central venous access through the external jugular vein in children submitted to bone marrow transplantation. Braz Arch Biol Technol. 2005;48(1):41-4.

6. Mandelbaum SH, Di Santis EP, Mandelbaum MHS. Cicatrização: conceitos atuais e recursos auxiliares. An Bras Dermatol. 2003;78(5):525-40.

\section{CONCLUSÃO}

Hoje há no mercado uma imensa gama de coberturas e agentes tópicos. Cabe ao enfermeiro analisar criticamente o custo/benefício de suas ações visando o sucesso do tratamento. Para o tratamento da ferida do caso relatado, a seleção dos agentes: Alginato de Cálcio e Dersani ${ }^{\circledR}$ foi realizada com base na avaliação das características da ferida e evidências científicas, relacionadas aos produtos, apresentadas na literatura, aliado a um custo relativamente baixo. Como o tratamento foi realizado por uma equipe composta por três enfermeiras, a comunicação foi bastante efetiva e se deu através da discussão do caso entre os membros desta equipe e a paciente, se iniciou com a escolha do tratamento e perdurou durante todo o processo, até a alta. As evoluções de enfermagem diárias acompanhados de registro fotográfico do processo deram um subsídio valiosíssimo para o acompanhamento da evolução da ferida e posteriormente a idealização deste artigo.

7. Dantas SRPE. Aspectos históricos do tratamento de feridas. In: Jorge SA. Abordagem multiprofissional do tratamento de feridas. São Paulo: Atheneu; 2003. p. 3-6.

8. Guidugli-Neto J. Elementos de patologia geral. São Paulo: Grupo GEN; 1997.

9. Dealey C. Cuidando de feridas: um guia para as enfermeiras. São Paulo: Atheneu; 2001. p. 49-65.

10. Ferreira E, Lucas R, Rossi LA, Andrade D. Curativo do paciente queimado: uma revisão de literatura. Rev Esc Enferm USP. 2003;37(1):44-51.

11. Conselho Regional de Enfermagem de Minas Gerais (CORENMG). Deliberação 65/00. Dispõe sobre as competências dos profissionais de enfermagem na prevenção e tratamento das lesões cutâneas [Internet]. Belo Horizonte; 2000 [citado 2009 nov. 22]. Disponível em: http://www.corenmg.gov.br/sistemas/app/ web200812/interna.php?menu=0\&subMenu $=2 \&$ prefixos $=65$

12. Conselho Federal de Enfermagem (COFEN). Resolução COFEN 311/2007. Código de Ética dos Profissionais de Enfermagem [Internet]. Brasília; 2007 [citado 2009 nov. 22]. Disponível em: http://www.portalcofen.gov.br/sitenovo/node/4394
Correspondência: Luciana Mendes Bruzi Rua Síria Thebit, 82 - Morada do Rio CEP 33030-710 - Santa Luzia, MG, Brasil 